Meta

Journal des traducteurs

Translators' Journal

\title{
Mini-glossaire trilingue (Français-Anglais-Italien) de la restauration des peintures
}

Jocelyne Boyer

Volume 16, numéro 4, décembre 1971

URI : https://id.erudit.org/iderudit/002136ar

DOI : https://doi.org/10.7202/002136ar

Aller au sommaire du numéro

Éditeur(s)

Les Presses de l'Université de Montréal

ISSN

0026-0452 (imprimé)

1492-1421 (numérique)

Découvrir la revue

Citer cet article

Boyer, J. (1971). Mini-glossaire trilingue (Français-Anglais-Italien) de la restauration des peintures. Meta, 16(4), 224-231.

https://doi.org/10.7202/002136ar d'utilisation que vous pouvez consulter en ligne.

https://apropos.erudit.org/fr/usagers/politique-dutilisation/ 


\section{MINI-GLOSSAIRE TRILINGUE (FRANÇAIS-ANGLAIS-ITALIEN) DE LA RESTAURATION DES PEIN'TURES}

Dans ce petit glossaire, nous avons préféré nous limiter à quelques termes employés dans le domaine de la restauration pour désigner les maladies des peintures et les modes de restauration des tableaux.

Les techniques de restauration variant d'un pays à l'autre, il nous a été parfois impossible de trouver le terme précis pour traduire une réalité donnée, comme dans le cas de «table chauffante», procédé de «doublage » utilisé en Amérique et en France mais dont l'emploi n'est pas encore répandu en Italie.

Nous remercions la direction et le personnel du Laboratoire d'examen des peintures et de l'Atelier de restauration du Louvre, ainsi que l'Institut de restauration de Rome et le Centre international d'Études supérieures pour la conservation et la restauration des biens culturels (C. I. E. S.) qui nous ont généreusement apporté leur aide et leurs conseils éclairés dans notre recherche terminologique multilingue.

Avant d'aborder le glossaire proprement dit, rappelons brièvement de quoi se compose une peinture. Il y a tout d'abord le «support», panneau ou toile. On recouvre celui-ci d'une "préparation » ou encollage, substance inerte (gypse, craie, blanc de plomb ou de titane) mêlée à la colle et qui a pour but de protéger la toile contre l'action chimique de l'huile. Puis vient la « couche picturale » qui consiste en un agrégat de pigments liés par un agglutinant : huile, résine ou gomme, auquel on a ajouté un peu de siccatif pour hâter le séchage et une très faible quantité de diluant (essence) pour rendre le mélange facile à appliquer. Lorsque le tableau est sec, on le recouvre enfin d'une couche de «vernis 》 qui protège la peinture en lui donnant de l'éclat. C'est ce vernis qui s'altère et s'assombrit avec l'âge et que l'on doit remplacer plusieurs fois au cours des siècles, même s'il donne à l'œuvre une certaine patine fort prisée par quelques peintres et collectionneurs. 
Schéma des couches stratifiées constiturives d'une peinture sur panneau (section verticale) *

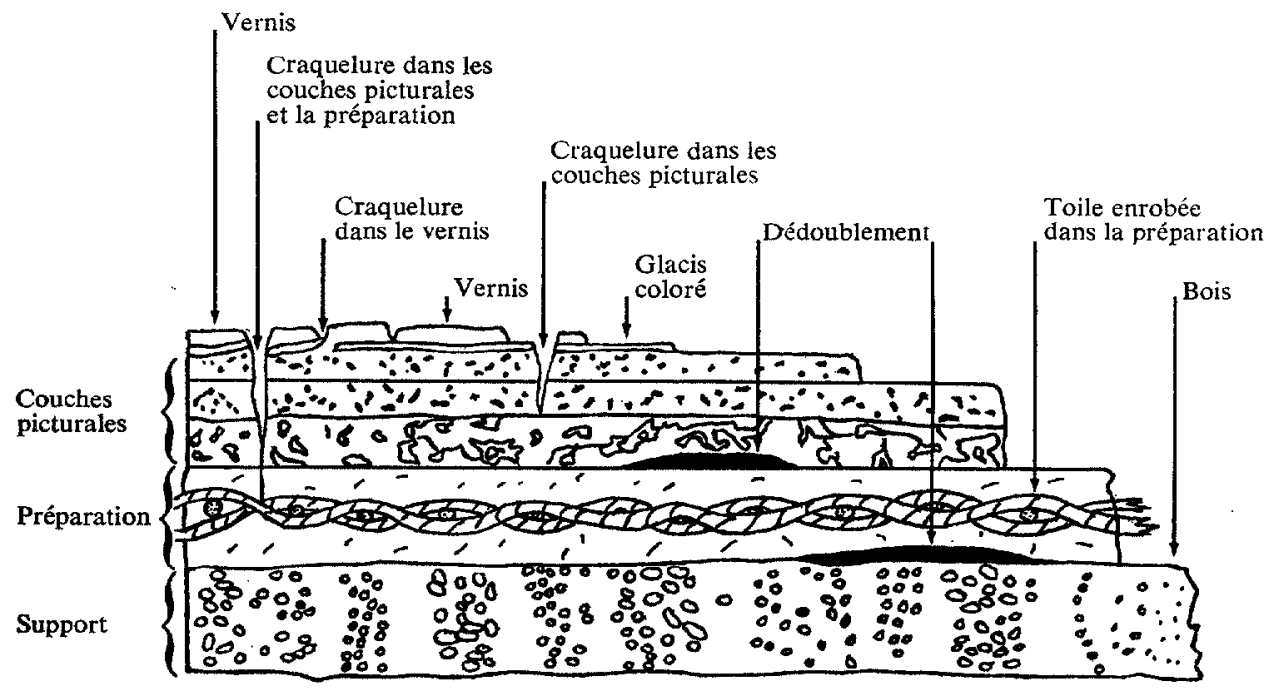

* D'après Plenderleith, la Conservation des antiquités et des auvres d'art.

ALLÉGEMENT : Enlèvement partiel du vernis, de façon à laisser intacte la très mince pellicule qui a pu pénétrer dans la couche picturale lors du vernissage. On se sert d'un dissolvant à évaporation très rapide, acétone ou pétrole dilué, pour ne pas attaquer la peinture. Le restaurateur dispose d'une quarantaine de produits, dissolvants actifs et dissolvants doux, qui peuvent être combinés entre eux (voir l'article «nettoyage»).

angl. : Partial removal of varnish : Il n'existe pas de terme consacré pour rendre « allégement». L'anglais emploie des périphrases explicatives : «To reduce the thickness of varnish ${ }^{1}$, "To fine down the varnish layer ${ }^{2}$ ».

ital. : Allegerimento : «Assottigliamento dello strato di vernice ${ }^{3} »$.

BLEUISSEMENT : Espèce de voile bleuâtre qui se forme sur le vernis de protection des tableaux. Ces vernis à l'essence pure (cristal dammar, mastic) ont l'avantage de s'enlever rapidement au cours des dévernissages successifs, mais ils sont très sensibles à l'humidité avant et après le vernissage. "Les changements de saison sont particulièrement favorables à l'évolution du «bleuissement des vernis vers une altération infiniment plus grave, qui risque de dégrader non seulement la couche superficielle du vernis mais la peinture elle-même, le « chancis $^{4} »$.

1. U.N.E.S.C.O. \& I.C.O.M., The Care of Paintings, p. 146.

2. R. Marijnissen, Dégradation, conservation, restauration de l'œuvre d'art, vol. II, p. 564.

3. C.I.E.S., Rome, traduction du Glossaire du Louvre, p. 1.

4. Xavier de L'Anglais, la Technique de la peinture à l'huile, p. 361. 
angl. : Bloom : «This word has been used to describe the dull bluishwhite appearance that develops in a transparent film and leads to a cloudiness. Damp is certainly a major factor in the "blooming » of varnish ${ }^{5}$.

ital. : Appannamento o appannatura 6 .

BOUCHAGE DES TROUS ou MASTICAGE : Opération qui consiste à remplir les trous causés par les vers ou par les écailles tombées de la couche picturale à l'aide d'un mastic composé le plus souvent de blanc à la colle qui est ensuite poncé avant retouche. Les restaurateurs français préfèrent le vocable " masticage », plus concis et plus descriptif.

angl. : Filling, stopping : Les avis sont partagés. À I'U. N. E. S. C. O. et à I'I. C. O. M., on emploie plutôt filling, et R. Marijnissen préfère stopping. Nous optons pour filling, d'après filler : matière de remplissage.

ital. : Stuccatura.

BOURSOUFLURE : Sorte de gonflement, de déformation de la préparation et de la couche picturale se détachant du support par morceaux. Cette maladie est plus fréquente dans les peintures sur panneaux que dans les peintures sur toile. Les tableaux italiens exécutés sur bois tendre (peuplier) en souffrent particulièrement. Les boursouflures peuvent affecter diverses formes : lorsqu'elles sont rondes et causées par la chaleur, on les appelle « cloques ». Maintenant, grâce à la table chauffante, " on peut chauffer le tableau jusqu'à ce que la peinture devienne malléable et l'on fait progressivement le vide sous la couche picturale, résorbant toutes les «boursouflures » d'un seul coup ${ }^{7} \gg$.

angl. : Blistering : «Occurs in oil paintings, involving the paint film alone or both the paint film and the ground. Results generally from moisture present in the ground or support or because the picture has been subjected to heat (sun or fire) ${ }^{8}$. " Le terme anglais a une plus grande extension car il traduit à la fois «boursouflure » et « cloque ».

ital. : Bolle di pittura.

CHANCIS ou CHANCI, dont l'orthographe varie avec les auteurs : \& Le chancis fait suite au bleuissement. À ce stade, la résine du vernis reprend sa forme amorphe d'origine et devient pulvérulente. La peinture se recouvre d'un voile blanc nébuleux qui cache les détails du dessin et peut rendre l'œuvre méconnaissable. Une application de solvant cristallisera de nouveau la résine et rendra à la toile sa condition primitive. Causes : air humide ou projection d'eau ${ }^{9}$. » Lorsque le chancis prend la forme de champignons minuscules envahissant les fibres, le « transfert » s'impose.

angl. : Mould (Madeleine Hours, Louvre) ou Mouldy (Office international des musées).

5. H. J. Plenderleith, The Conservation of Antiquities and Works of Art, p. 172.

6. Grande dizionario della lingua italiana, vol. I, p. 547-548.

7. Studies in Conservation, périodique, vol. VIII, no 2 , mai 1963, p. 63

8. C. D. Clarke, Pictures, their Preservation and Restoration, p. 139.

9. M. Aubert, Louvre, Atelier de restauration. 
ital. : Sbianchamento (blanchissement) lorsqu'il désigne l'apparence ou Intanfito (moisi) lorsqu'il se rapporte à la cause.

CUVETTE(S) : Maladie de la couche picturale fréquente dans les grandes toiles du XVII ${ }^{e}$ siècle. Les lèvres des craquelures déjà formées se relèvent et la couche picturale prend l'aspect d'une série de petites cuvettes, sans toutefois qu'il y ait détachement. Certains restaurateurs du Louvre les ont baptisées \& écailles en tuiles ».

angl. : Cupping : "Concavity of the islands of paint surrounded by crackles with raised edges ${ }^{10}$. $\gg$ Comme dans la plupart des cas, le nom de la maladie est rendu en anglais par le substantif avec terminaison en ing.

ital. : Scodelline.

DÉDOUBLEMENT : Séparation horizontale qui se produit entre les différentes pellicules picturales ou entre la couche picturale et le support. Cet accident est causé par les changements extrêmes de l'humidité relative de l'atmosphère et est fréquent dans les peintures anciennes dont le liant a perdu de l'élasticité. On y remédie par le «transfert» ou par l'imprégnation à la cire résine avec utilisation de la «table chauffante ».

angl. : Cleavage : L'extension du terme anglais est plus vaste car il désigne l'accident lui-même et l'espace vertical, la poche d'air qui en résulte : « $A$ division, parallel to the surface in the paint, ground or support, or between two of these layers ${ }^{11} . » ~ " P o c k e t s$, air bells, looseness, or other separation of the ground. parallel to the surface and either below or in the ground film ${ }^{12}$. 》

ital. : Sdoppiamento : Traduit littéralement « dédoublement».

DEXTRE (à) : Terme employé exclusivement au Louvre. Il a été emprunté à l'héraldique pour désigner, sur la toile, le côté qui se trouve à la gauche de l'observateur et à la droite du ou des personnages représentés. Terme discutable. En effet, comment justifier cette expression dans l'étude de paysages ou d'abstractions? angl. : Dexter : "In heraldy, the user's right side and the viewer's left side of a shield. In painting, may be used to designate the left side of the observer ${ }^{13}$.» ital. : Sinistra (a) : L'italien rétablit la réalité : «à gauche».

Remarque : Les mêmes particularités s'appliquent à l'expression inverse : «à sénestre », sinister, a destra.

EMBU : "Lorsqu'une peinture est exécutée sur un fond qui n'est pas assez sec, l'huile est aspirée en plaques par les couches inférieures et il en résulte des aspects ternes, nébuleux, opaques qui déforment complètement les valeurs des couleurs. Ce terme ne s'applique qu'à la peinture. Pour désigner les plaques ternes d'un vernis, on dira plutôt des «mats » ou des « matités ${ }^{14}$ ».

angl. : Sinking in : (The dullness on the surface of an oil painting which results

10. M. C. Bradley, The Treatment of Pictures, "Glossary ».

11. Ibid.

12. Ibid.

13. R. G. Haggar, A Dictionary of Art Terms, p. 109.

14. $\mathbf{M}^{\text {ues }}$ Chocqueel et Toupet, $\mathbf{M}^{\mathrm{me}}$ Nazat, $\mathrm{H}$. Linard, Atelier de restauration, Louvre. 
from the colors sinking in. This is due to the oil being absorbed by the ground or by an underlayer of paint.) En anglais, sinking in est employé indifféremment pour désigner les plaques ternes du vernis ou des couleurs. On notera ici l'addition de la postposition in au substantif.

ital. : Appannato.

NETTOYAGE : Il faut distinguer entre le nettoyage en profondeur ou « dévernissage », opération par laquelle on enlève, au moyen d'un scalpel ou d'un dissolvant, les vernis anciens ou autres incrustations qui peuvent assombrir un tableau, et le nettoyage superficiel appelé au Louvre «bichonnage » et ailleurs 《 décrassage », " dépoussiérage », qui est une toilette superficielle faite à un tableau en bon état, sans qu'il y ait restauration. Le terme «bichonnage» nous semble bien rendre la minutie, les soins attentifs que requiert cette opération délicate.

angl. : Cleaning : (The complete removal of old, hard and dirty varnish is called «stripping» by the restorers, when retouching and revarnishing are included.)

«In the United States, cleaning may also be spoken of as « dabbing », an inadequate and non-descriptive term which should be discarded for «surface cleaning》 (when it involves\}, removal of superficial dirt but not of varnish ${ }^{15}$. »

ital. : Pulitura : Sverniciatura, pour le dévernissage, et pulitura superficiale pour le bichonnage.

PEAU DE CRAPAUD : Maladie de la couche picturale connue également sous le nom de "ridage » et due à un défaut de préparation ou à une exécution fautive. La peinture grasse, appliquée sur un fond gras plutôt que sur une préparation maigre, se plisse et se contracte en séchant pour former une série de petites saillies qui donnent à la couche picturale l'apparence d'une peau de crapaud. Phénomène fréquent dans les peintures du xix ${ }^{\mathrm{e}}$ siècle.

angl. : Alligatoring : "Defect appearing as a uniform cracking of the paint film shrinking from the ground and leaving spaces between each cracked area. It is due to improper building up of the paint film and improperly prepared ground on the support ${ }^{16} . \gg$ Chose étrange, le crapaud se mue en alligator. Bon exemple de modulation.

ital. : Pittura increspata.

PLAGE (de repeints ou autres) : Substantif habituellement employé au pluriel en langage de restauration. Ce terme est emprunté au vocabulaire de la photographie où il désigne une surface, par opposition à des points. La surface d'une peinture peut présenter des «plages» brillantes ou mates, quand il s'y trouve «repeints» ou « embu».

angl. : Area : Le terme anglais a une extension très vaste et n'est pas aussi imagé que le terme français.

ital. : Zona : Même remarque que pour le mot anglais.

15. C. D. Clark, Pictures, their Restoration and Preservation, p. 166-167.

16. Ibid., p. 137. 
POINTILLAGE : En restauration, petites retouches. «On pose l'un à côté de l'autre une multitude de petits points de couleur, absolument comme s'il s'agissait de peindre en miniature ${ }^{17}$. 》Ce procédé diffère du « repiquage » dans lequel les touches posées sont infiniment petites, comme des têtes d'épingle.

angl. : Dotting : Même extension qu'en français.

ital. : Puntini : Du français, « petits points ».

RÉGÉNÉRATION : Procédé de restauration mis à l'honneur au XIX ${ }^{e}$ siècle par le chimiste allemand Von Pettenkofer. On remédie à l'altération des vernis en les régénérant plusieurs fois au moyen de vapeurs d'alcool. « $\mathrm{Ce}$ procédé, extrêmement délicat, ne peut être appliqué que par des restaurateurs très expérimentés. Certaines peintures réagissent très rapidement à ce traitement et seraient détruites si elles étaient soumises trop longtemps à l'action des vapeurs d'alcool ${ }^{18}$. » À cause de cet inconvénient, le procédé est de moins en moins employé. Les restaurateurs préfèrent régénérer le vernis avec une brosse très souple trempée dans l'alcool et essorée. Les couleurs s'en trouvent avivées et le vernis reprend son éclat (M. Roullet, Atelier de restauration, Louvre).

angl. : Regeneration : «Reforming of surface coating by exposure to solvent vapor ${ }^{19}$.

ital. : Ringiovanimento (delle vernici).

RENTOILAGE ou DOUBLAGE : Opération qui consiste à coller une toile neuve au dos de l'originale. "L'emploi abusif de l'expression « rentoilage » a pu faire croire au profane que chaque fois qu'on rentoile une peinture on supprime la toile originale; mais l'ancienne toile subsiste, et l'opération consiste simplement à la consolider par une nouvelle toile, à la doubler ${ }^{20}$. 》 Pour éviter toute confusion, on lui préférera le terme "doublage», couramment employé en Allemagne. La technique ancienne de doublage à la colle est de plus en plus délaissée en faveur de la technique hollandaise à base de cire et de résine.

angl. : Relining : "The lining or mounting of a picture with its old canvas upon a new linen canvas support ${ }^{21}$.

ital. : Rintelaggio.

REPEINT : «Partie d'un tableau qui a été repeinte soit par l'auteur, soit par un restaurateur. » Ne pas confondre avec « repentir» (pentimento) qui est « un changement, une correction apportée par l'auteur à son œuvre en cours d'exécution (tandis que), le repeint est fait après coup ${ }^{22}$ ».

angl. : Repaint : (A part of a picture repainted by a restorer.) Le terme inpainting est plus vaste car il englobe toutes les retouches : « repiquages », "rigatini », «pointillages », etc.

ital. : Ridipintura.

17. C. Dalbon, Traité technique et raisonné de la restauration des tableaux, p. 103-104.

18. Mouseion, vol. 41-42, no 11, 1938, p. 120.

19. M. C. Bradley, The Treatment of Pictures, « Glossary *.

20. Mouseion, vol: $41-42, \mathrm{n}^{\circ} 11,1938$, p. 229.

21. R. G. Haggar, A Dictionary of Art Terms, p. 285.

22. Le Petit Robert, p. 1521. 
RIGATINI : État de restauration visible de près et imperceptible à distance, consistant en la juxtaposition de couleurs pures par petits traits fins. C'est l'œil qui opère le mélange.

angl. : Rigatini : "This consists in completely reconstructing the missing parts, perfectly matching form and colour, but in fine separate stripes or hatching, which blend into a coherent effect when seen from a normal viewing distance ${ }^{23}$. 》

ital. : Rigatini : Le terme italien, comme c'est souvent le cas en art, est employé dans les trois langues.

SONDAGE : Étape préparatoire à la restauration qui consiste en dévernissage ou «allégement » par petits carrés, pour déterminer la nature et l'état du vernis à remplacer.

angl. : Trial cleaning : Certains restaurateurs emploient le mot probe, à extension plus large mais qui a l'avantage d'être concis.

ital. : Prova di pulitura ${ }^{24}$.

TABLE CHAUFFANTE : Appareil employé pour l'imprégnation et le «doublage» des peintures et qui consiste en une plaque de métal chauffée en plusieurs endroits par des résistances électriques pour obtenir un degré de chaleur contrôlable. Elle comporte quatre prises qui aspirent l'air sous la table et font le vide sous la toile. On l'emploie désormais de préférence au fer à repasser, évitant ainsi d'écraser les empâtements (H. Linard, Atelier de restauration, Louvre).

angl. : Hot table : «A table with a heatable upper surface generally of metal, used in the lining of canvas picture supports with thermoplastic adhesives ${ }^{25}$. 》

ital. : L'usage de la «table chauffante » n'étant pas répandu en Italie, il n'existe aucun terme pour désigner cette réalité. On évitera l'emploi de tavola calda à cause de l'expression identique employée pour désigner un buffet chaud en Italie.

TRANSFERT : Procédé extrêmement délicat inventé au XVII ${ }^{\mathrm{e}}$ siècle par un restaurateur français nommé Picault. Dans les cas de dégradation grave du support ou lorsque la couche picturale tend à se séparer de la préparation, il y a lieu de procéder à un transfert. C'est ainsi que l'on nomme la transposition de la couche peinte tout entière sur un autre support. Selon les auteurs et les restaurateurs, l'opération prend indifféremment le nom de «transfert 》 ou de «transposition ». angl. : Transfer : «Major operation which means removing the original support and replacing it by a new one ${ }^{26}$. 》

ital. : Trasporto : A très grande extension.

JOCELYNE BOYER

23. H. Ruhemann, The Cleaning of Paintings, p. 258.

24. M $M^{\text {me }}$ Kruse, Institut de restauration, Rome.

25. C.I.E.S., Rome, traduction du Glossaire du Louvre.

26. M. Hours-Miédan, The Secrets of the Great Masters, p. 124. 


\section{BIBLIOGRAPHIE}

Bradley, M. C., The Treatment of Pictures, « Glossary », Cambridge, Art Technology, 1950.

CANADIAN INDUSTrIEs LTD., Fiches linguistiques, Montréal.

C.I.E.S., Rome, traduction du Glossaire du Louvre.

Clarke, C. D., Pictures, their Preservation and Restoration, Butler (Maryland), The Standard Arts Press, $1959,270 \mathrm{p}$.

Dalbon, Charles, Traité technique et raisonné de la restauration des tableaux, Paris, L. H. May, $1898,146 \mathrm{p}$.

DE L'ANglaIs, Xavier, la Technique de la peinture à l'huile, Paris, Flammarion, 1959, $421 \mathrm{p}$.

Grande dizionario della lingua italiana, Turin, Unione tipografico, 1961-1966.

GuIllerme, J., l'Atelier du temps, Paris, Hermann, 1964, 246 p.

HAggar, Reginald G., A Dictionary of Art Terms, Londres, Oldbourne Press, 1962, 416 p.

Hours-Mı́́dan, Madeleine, les Secrets des chefs-d'auvre, Paris, Laffont, 1964, 215 p.

-, The Secrets of the Great Masters, Toronto, Longmans Canada Ltd.

Marijnissen, R., Dégradation, conservation, restauration de l'auvre d'art, 2 vol., Bruxelles, Arcade, 1967.

Office international des musées, Mouseion/Museum, Paris, U. N. E. S. C. O.

-, Manuel de la conservation et de la restauration des peintures, "Lexique trilingue ", Paris, Institut int. de coop. intellectuelle, 1939, $310 \mathrm{p}$.

Plenderleith, H. J., The Conservation of Antiquities and Works of Art, New York, Oxford Press, $1956,400 \mathrm{p}$.

RunemanN, H., The Cleaning of Paintings, Londres, Faber \& Faber, 1968, $508 \mathrm{p}$.

Studies in Conservation, Farmers Hall, Aberdeen, Ecosse, The Aberdeen University Press Ltd.

U. N. E. S. C. O. \& I. C. O. M., The Care of Paintings, compilation d'articles parus dans la revue Museum, vol. III, $\mathrm{n}^{\circ \mathrm{s}} 2$ et 3, 1950; vol. IV, $\mathrm{n}^{\circ} 1,1951$. 